\title{
A Probabilistic Model for Correspondence Problems Using Random Walks with Restart
}

\author{
Tae Hoon Kim, Kyoung Mu Lee, and Sang Uk Lee \\ Dept. of EECS, ASRI, Seoul National University, 151-742, Seoul, Korea \\ thkim@diehard.snu.ac.kr, kyoungmu@snu.ac.kr, sanguk@ipl.snu.ac.kr
}

\begin{abstract}
In this paper, we propose an efficient method for finding consistent correspondences between two sets of features. Our matching algorithm augments the discriminative power of each correspondence with the spatial consistency directly estimated from a graph that captures the interactions of all correspondences by using Random Walks with Restart (RWR), one of the well-established graph mining techniques. The steadystate probabilities of RWR provide the global relationship between two correspondences by the local affinity propagation. Since the correct correspondences are likely to establish global interactions among them and thus form a strongly consistent group, our algorithm efficiently produces the confidence of each correspondence as the likelihood of correct matching. We recover correct matches by imposing a sequential method with mapping constraints in a simple way. The experimental evaluations show that our method is qualitatively and quantitatively robust to outliers, and accurate in terms of matching rate in various matching frameworks.
\end{abstract}

Keywords: Random Walks with Restart, feature correspondence, sequential matching.

\section{Introduction}

Feature correspondence is one of the fundamental problems of computer vision and lies at the core of many applications including 3D reconstruction and object recognition. To solve this problem, several approaches [9] [3] [7] have been proposed. One approach is to formulate the matching problem as an integer quadratic programming, like 9 3. However, the method of Maciel et al. 9] is based on a non-optimal minimization technique with non-polynomial complexity, and the method by Berg et al. 3] is more suitable only for the case of allowing several features to match the same feature. The other one is to use a spectral technique for finding consistent correspondences between two sets of features, like [7. Leordeanu et al. 7] used the principal eigenvector of the pairwise affinity matrix corresponding to its largest eigenvalue as the information about how strongly the matching candidates belong to the optimal set. However, their results depend mainly on the properties of the affinity matrix such as the number of links adjacent to each correspondence in local neighborhood system. Moreover, it is difficult to control the scale of each individual appearance-based matching score compared with the geometric affinities in a principal manner.

H. Zha, R.-i. Taniguchi, and S. Maybank (Eds.): ACCV 2009, Part III, LNCS 5996, pp. 416-425, 2010. (C) Springer-Verlag Berlin Heidelberg 2010 
In this paper, we introduce an efficient probabilistic method for solving various correspondence problems. We propose to estimate the likelihood that each candidate corresponds to the consistent correspondences between two sets of features by the Random Walks with Restart (RWR) 6] 11, one of the wellestablished graph mining techniques. Namely, this likelihood of one matching candidate is defined as the weighted sum of all the steady-state probabilities between that candidate and other candidates in the RWR framework. We finally recover the optimal matches from the estimated likelihoods by simply adopting a sequential method with mapping constraints, as in the work of Leordeanu et al. 7]. Our matching framework has various advantages over [7] as follows. First, since RWR, similarly to the graph-based semi-supervised learning [13, has the ability to estimate the global relevance between pairs of correspondences by the local affinity propagation, our proposed likelihood of each correspondence represents its confidence by considering global interactions among highly consistent correspondences. Second, we easily define the combination of two properties: the discriminative power of each individual correspondence and the pairwise geometric affinities, in a probabilistic framework. Finally, our approach can be applied to various correspondence problems between points, regions, or interest points 8] 10] 12. In case of non-discriminative features like points, the only pairwise geometric information helps in finding the correct correspondences. When discriminative features like regions or interest points are employed, both the geometric relations and each individual appearance-based matching score can be utilized for improving the performance.

\section{Proposed Algorithm}

Feature matching is to find the optimal set $L^{o p t}$ of well-matched pairs in a candidate set $L=\left\{l_{n}\right\}_{n=1, \ldots, N}$ of the initial feature correspondences $l_{n}=\left(x_{n}, x_{n^{\prime}}\right)$, where the features $x_{n}$ and $x_{n^{\prime}}$ are extracted in two sets $X$ and $X^{\prime}$ respectively. Let $e$ be the event of a candidate be matched. We intend to estimate the likelihood $\theta_{n}=p\left(l_{n} \mid e\right)$ that the candidate $l_{n}$ is a correct match. We finally recover the optimal matches $L^{o p t}$ from the total likelihoods $\left\{\theta_{n}\right\}_{n=1, \ldots, N}$ by simply imposing a sequential method with mapping constraints.

\subsection{Likelihood Estimation}

The likelihood $\theta_{n}$ can be obtained by

$$
\theta_{n}=\sum_{l_{k} \in \bar{L}} p\left(l_{n} \mid l_{k}, e\right) p\left(l_{k} \mid e\right)=\sum_{l_{k} \in \bar{L}} \pi_{n}^{k} \eta_{k},
$$

where $\bar{L} \subset L$ is a set of the seed correspondences. All candidates are generally used as seeds, $\bar{L}=L$. However, if there are many outliers with very similar appearances, it is more effective to use only a few correspondences, that are likely to exist in the optimal set $L^{o p t}$, as seeds. Each likelihood $\theta_{n}$ is modeled by a 
mixture of distributions $\pi_{n}^{k}=p\left(l_{n} \mid l_{k}, e\right)$ from each seed $l_{k} \in \bar{L}$ which has an initial seed distribution $\eta_{k}=p\left(l_{k} \mid e\right)$. The distribution $\pi_{n}^{k}$ indicates the geometric relevance score between a candidate $l_{n}$ and a seed $l_{k}$. In this work, we propose to use the RWR steady-state probability based on the pairwise local affinities, as similar to the segmentation framework of Kim et al. 6]. Compared with traditional graph distances (such as shortest path, maximum flow), this steady-state probability can capture the whole relationship between two candidates. The seed distribution $\eta_{k}$ corresponds to the weight of the seed $l_{k}$. Namely, it means how well the features' descriptors of the seed $l_{k}$ match to each other. In brief, our matching algorithm combines the discriminative power of each correspondence with the global geometric interactions in a probabilistic framework. Now, we describe our proposed distributions $\pi_{n}^{k}$ and $\eta_{k}$ in detail.

Estimating Pairwise Relationship of Correspondences. Let us consider an undirected weighted graph $G=(L, E)$, where each node $l_{n} \in L$ uniquely identifies a feature correspondence, and each edge $e_{n m} \in E$ spanning between two nodes $l_{n}, l_{m} \in L$ is determined by the neighborhood system. Each weight $w_{n m} \in W$ is assigned to the edge $e_{n m}$, and measures how compatible the features $\left(x_{n}, x_{m}\right)$ in a set $X$ are with $\left(x_{n^{\prime}}, x_{m^{\prime}}\right)$ in the other set $X^{\prime}$ by the computation of the geometric consistency between two correspondences $l_{n}=\left(x_{n}, x_{n^{\prime}}\right)$ and $l_{m}=\left(x_{m}, x_{m^{\prime}}\right)$. The affinity matrix $\mathbf{W}=\left[w_{n m}\right]_{N \times N}$ may be differently designed according to various applications. We will introduce various pairwise affinity models in Section 3 in more detail.

Now, we propose to use the steady-state probability of RWR, that captures the whole relationship between a candidate $l_{n}$ and a seed $l_{k}$ in this graph $G$, via the geometric affinity $\pi_{n}^{k}$ in (11). Suppose that a random walker starts from a seed $l_{k}$, and iteratively transmits to its neighborhood with the probability that is proportional to the edge weight between them. Also at each step, it has a restarting probability $\lambda$ to return to the seed $l_{k}$. After convergence, we obtain the steady-state probability that the random walker will finally stay at the node $l_{n}$. Since this steady-state probability considers all possible paths between the two correspondences $l_{n}$ and $l_{k}$, it is suitable measure for the distribution $\pi_{n}^{k}$ in (11). By setting a vector $\boldsymbol{\pi}^{k}=\left[\pi_{n}^{k}\right]_{N \times 1}$, RWR can be formulated as follows [6] [11.

$$
\boldsymbol{\pi}^{k}=(1-\lambda) \mathbf{P} \boldsymbol{\pi}^{k}+\lambda \boldsymbol{h}^{k},
$$

where $\boldsymbol{h}^{k}=\left[h_{n}^{k}\right]_{N \times 1}$ is the seed indicating vector with $h_{n}^{k}=1$ if $n=k$ and 0 otherwise, and the transition matrix $\mathbf{P}$ is the adjacency matrix $\mathbf{W}$ row-normalized: $\mathbf{P}=\mathbf{D}^{-1} \times \mathbf{W}$, where $\mathbf{D}=\operatorname{diag}\left(d_{1}, \ldots, d_{N}\right), d_{n}=\sum_{m=1}^{N} w_{n m}$. The random walker positioned at each correspondence $l_{n}$ is returned with the restarting probability $\lambda$. With smaller $\lambda$, the current state becomes more emphasized and more propagated to its neighborhoods. We empirically set $\lambda=0.01$ for all experiments.

Estimating Initial Seed Weights. A seed weight $\eta_{k}$ in (11) means how well matched both features of the seed correspondence $l_{k}$ are. Let $\hat{\boldsymbol{\eta}}=\left[\hat{\eta}_{k}\right]_{N \times 1}$ be the seed weight vector with $\hat{\eta}_{k}=\eta_{k}$ if $l_{k} \in \bar{L}$ and 0 otherwise. This vector $\hat{\boldsymbol{\eta}}$ can be differently designed according to the feature types for the specific applications. 
In case of non-discriminative features such as points, there are no initial scores on the individual correspondences. In this case, we use the principal eigenvector of the pairwise affinity matrix $\mathbf{W}$ as in the work of Leordeanu et al. 7]. Their work is to find the optimal solution $\boldsymbol{u}^{*}=\operatorname{argmax}_{\boldsymbol{u}}\left(\boldsymbol{u}^{T} \mathbf{W} \boldsymbol{u}\right)$ that maximizes the inter-cluster score $S=\sum_{l_{n}, l_{m} \in L} w_{n m}$. By the Raleigh's ratio theorem, this solution $\boldsymbol{u}^{*}$ is the principal eigenvector of $\mathbf{W}$. Since the eigenvector $\boldsymbol{u}^{*}$ denotes the unary scores that represent how strongly each correspondence belongs to the optimal set, it is defined as our seed weight vector $\hat{\boldsymbol{\eta}}=\boldsymbol{u}^{*}(|\hat{\boldsymbol{\eta}}|=1)$.

For discriminative features like regions or interest points [8] 10] 12, we use the following seed weight $\hat{\eta}_{k}$ based on the Euclidean distance between two features $x_{k}$ and $x_{k^{\prime}}$ of each seed $l_{k}$ with feature properties $v_{k}$ and $v_{k^{\prime}}$.

$$
\hat{\eta}_{k}=\left\{\begin{array}{cc}
\exp \left(-\left\|v_{k}-v_{k^{\prime}}\right\| / 2 \sigma_{w}^{2}\right) & l_{k} \in \bar{L} \\
0 & \text { otherwise }
\end{array}\right.
$$

where $\sigma_{w}$ is the variance of the total feature properties. In this work, all candidates are used as seeds, $\bar{L}=L$. Although each seed weight provides a proper information about how well the features' descriptors match, it does not give absolute confidence under difficult conditions such as nonrigid deformation, occlusion and illumination changes. So, if we can choose only a few reliable seeds instead of all candidates under uncertain conditions, they can help in finding better correspondences. For example, we can select the refined seeds $\bar{L}$ by adding an additional condition that the distance to the first nearest neighbor is closer than 0.7 times the distance to the second one. This constraint can be generally used for extracting more discriminative correspondences.

Overview of Our Probabilistic Model. Based on the RWR formulation in (2), the total likelihoods $\left\{\theta_{n}\right\}_{n=1, \ldots, N}$ in (1) can be written in a vector form, $\boldsymbol{\theta}=\left[\theta_{n}\right]_{N \times 1}$ such that

$$
\boldsymbol{\theta}=(1-\lambda) \mathbf{P} \boldsymbol{\theta}+\lambda \hat{\boldsymbol{\eta}}=\lambda(\mathbf{I}-(1-\lambda) \mathbf{P})^{-1} \hat{\boldsymbol{\eta}} .
$$

Since a small neighborhood system is used for the transition matrix $\mathbf{P}$, the matrix $(\mathbf{I}-(1-\lambda) \mathbf{P})$ in (4) is very large $(N \times N)$ but quite sparse. Therefore its inversion is practically feasible by efficient numerical methods. For example, the MATLAB division operator ' $\backslash$ ' (which we used in our experiments) serves as a very efficient tool in finding the inversion of such large sparse matrix.

\subsection{Finding Optimal Correspondences}

We finally choose the well-matched pairs $L^{o p t}$ from the estimated likelihoods $\boldsymbol{\theta}$ in (4). Algorithm 10scribes the selection procedure of correct matches, which is similar to the greedy algorithm of Leordeanu et al. 7. for finding the solution of the correspondence problem. We start by first selecting $l_{n}^{*}\left(=\operatorname{argmax}_{l_{m} \in L} \theta_{m}\right)$ that has the maximum likelihood as a correct correspondence, because it is the one we are most confident of being correct. Namely, we assign $l_{n}^{*}$ into $L^{\text {opt }}$. Next we have to reject all other candidates that are in conflict with $l_{n}^{*}$ by user-defined 


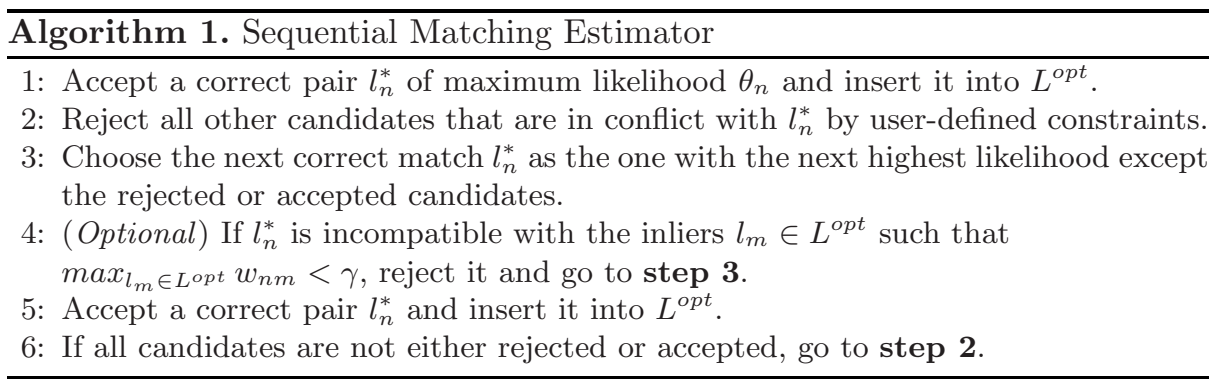

constraints. In our experiment, we reject all candidates of the form $\left(x_{n}, \cdot\right)$ or $\left(\cdot, x_{n^{\prime}}\right)$. Note that here the user could use different constraints. We accept the next correct match $l_{n}^{*}$ as the one that has the next highest likelihood among the remained candidates. In this accept step, we can optionally use the additional rejecting constraint in Algorithm 1,4, where the pairwise affinity score $w_{n m}$ is used for deciding the optimality of $l_{n}^{*}$. Since this constraint is based on the concept that the matches in $L^{o p t}$ have a tendency to smoothly change the local geometric deformation between the neighboring ones, we can easily find the optimal set $L^{o p t}$ regardless of the real sets with many outliers. We then continue by rejecting the pairs in conflict with the newly accepted one $l_{n}^{*}$. We repeat this procedure of accepting new match, until all candidates are either rejected or accepted. We finally produce the set $L^{o p t}$ of correct matches.

\section{$3 \quad$ Experiments}

We present experiments which demonstrate the performance of our approach to feature matching. In this section, we applied our algorithm to several different correspondence problems according to the feature types including Point Matching, Region Matching and Interest Point Matching. These problems have different affinity matrices $\mathbf{W}$ and seed weight vectors $\hat{\boldsymbol{\eta}}$ in (4).

Point Matching. We evaluate the robustness of our algorithm according to the deformation and the ratio of outliers to inliers on the task of finding correspondences between $2 \mathrm{D}$ point sets, like in [7. For quantitative evaluation, we study the case when the deformation noise is added by a Gaussian distribution with zero mean. We generate date sets of $2 \mathrm{D}$ model points $X$ by randomly selecting $N^{i}$ inliers in a given plane. We obtain the corresponding inliers in $X^{\prime}$ by disturbing independently the $N^{i}$ points from $X$ with white Gaussian noise $N\left(0, \sigma_{e}^{2}\right)$. The parameter $\sigma_{e}$ controls the degree of the deformations between two sets $X$ and $X^{\prime}$. Next we randomly add $N^{o}$ point outliers in $X$ and $X^{\prime}$, respectively, with the same random uniform distribution over the $x-y$ coordinates. The total number of points in $X\left(\right.$ or $\left.X^{\prime}\right)$ is $N^{p}=N^{i}+N^{o}$. The size of $L$ is $N=N^{p} \times N^{p}$ since all possible correspondences are used as candidates. In this experiment, we score the performance according to the variation of the deformation parameter $\sigma_{e}$ and the number of outliers $N^{o}$. 


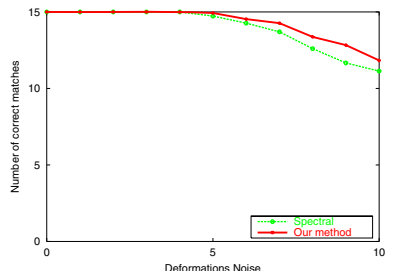

(a) $N^{i}=15, N^{o}=0$

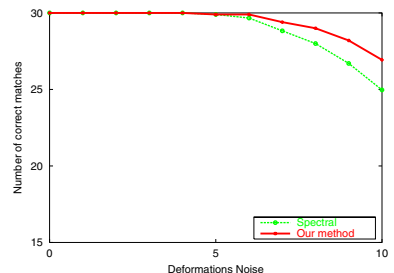

(b) $N^{i}=30, N^{o}=0$

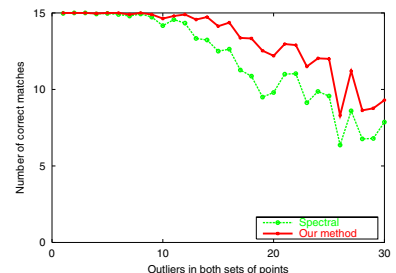

(c) $N^{i}=15, \sigma_{e}=2$

Fig. 1. Comparison of our method with spectral method ('Spectral') [7 for point matching. (a) and (b) show the number of the correct matches according to the variation of deformation noise $\sigma_{e}$. (c) compares the number of the correct matches according to the variation of the number of outliers $N^{o}$.

For estimating the likelihoods $\boldsymbol{\theta}$ in (4), the affinity matrix $\mathbf{W}=\left[w_{n m}\right]_{N \times N}$ is defined by the following pairwise score $w_{n m}$ between two candidates $l_{n}=$ $\left(x_{n}, x_{n^{\prime}}\right)$ and $l_{m}=\left(x_{m}, x_{m^{\prime}}\right)$ [7]. Note that a feature $x_{n}$ has the $x-y$ position vector $f_{n}$.

$$
w_{n m}=\left\{\begin{array}{cc}
4.5-\frac{\left(\mathcal{D}_{n m}-\mathcal{D}_{n^{\prime} m^{\prime}}\right)^{2}}{2 \sigma_{d}^{2}} & \text { if }\left|\mathcal{D}_{n m}-\mathcal{D}_{n^{\prime} m^{\prime}}\right|<3 \sigma_{d} \\
0 & \text { otherwise, }
\end{array}\right.
$$

where the functions $\mathcal{D}_{n m}=\left\|f_{n}-f_{m}\right\|$ and $\mathcal{D}_{n^{\prime} m^{\prime}}=\left\|f_{n^{\prime}}-f_{m^{\prime}}\right\|$ output the Euclidean distances between the pairs of points. The parameter $\sigma_{d}$ controls the sensitivity of the weight on deformations. The larger $\sigma_{d}$, the more deformations in the data we can accommodate, also the more pairwise relationships between wrong matches will get positive scores. We initially set $\sigma_{d}=5$. Since points $X$ and $X^{\prime}$ are non-discriminative and there are no information on the individual correspondences, the principal eigenvector of the affinity matrix $\mathbf{W}$ is used as the seed weights $\hat{\boldsymbol{\eta}}$. We finally produce the optimal set $L^{o p t}$ from these likelihoods $\boldsymbol{\theta}$ by Algorithm 1 without the optional step 4.

Fig. 1 shows the performance curves of our method vs. the spectral method [7. as we vary the deformative noise $\sigma_{e}$ from 0 to 10 (in steps of 1 ), the number of inliers $N^{i}$ from 15 to 30 , and the number of outliers $N^{o}$ from 1 to 30 (in steps of 1). Both methods ran on the same problem sets over 30 trials for each value of the varying parameter. We compared the performances of these two methods by counting how many matches agree with the ground truth. Compared with the spectral method [7] that depends mainly on neighborhood system to each candidate, our method increases the confidence of all correspondences by considering the whole relationship between the candidates.

Region Matching. In the task of finding region correspondences like 4, this experiment gives the average performances of the algorithms according to the variation of the region properties. For quantitative evaluation, we studied the case when the color noise was added by a Gaussian distribution with zero mean. We generated date sets of $N^{c} \times N^{c}$ grid regions $X$ with random colors, as shown 


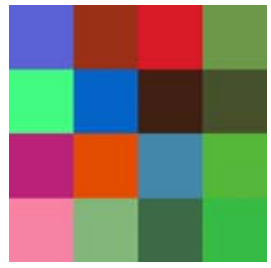

(a) $X$

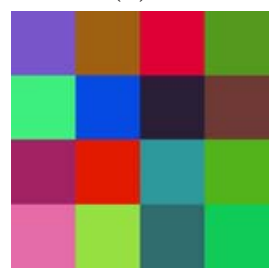

(b) $X^{\prime}$

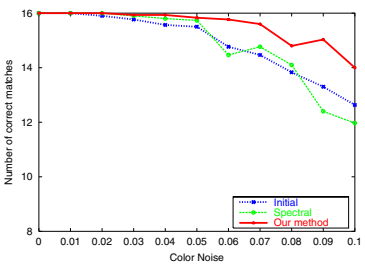

(c) $4 \times 4$ regions

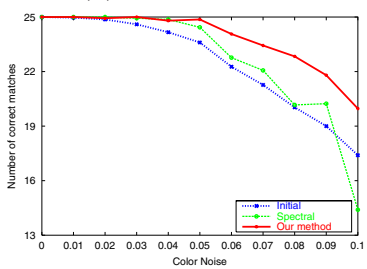

(d) $5 \times 5$ regions

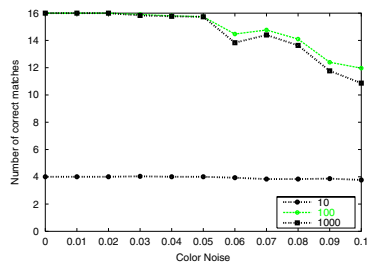

(e) $\kappa$

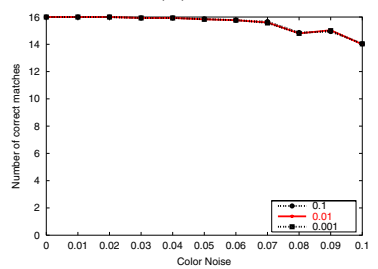

(f) $\lambda$

Fig. 2. Comparison of our method with the 'Initial' method and the 'Spectral' method 7] for region matching. (a) and (b) show the synthetic examples with $4 \times 4$ grid regions in $X$ and $X^{\prime}$, respectively. (c) and (d) compare the number of the correct matches according to the variation of the color noise $\sigma_{c}$. In case of $4 \times 4$ region matching, (e) and (f) check the sensitivities of the 'Spectral' method and 'Our' method according to the variation of the parameters $\kappa$ in (7) and $\lambda$ in (4), respectively.

in Fig. 2(a). We obtained the corresponding regions in $X^{\prime}$ by supporting the adjacency relations in $X$. We then disturbed independently the colors of the regions in $X^{\prime}$ with white Gaussian noise $N\left(0, \sigma_{c}^{2}\right)$, as shown in Fig. 2(b). The parameter $\sigma_{c}$ controls the degree of the color variance between two sets $X$ and $X^{\prime}$. The total number of regions in $X$ is $N^{p}=N^{c} \times N^{c}$. The size of candidates $L$ is $N^{p} \times N^{p}$, since all possible combinations were used as candidates. In this experiment, we scored the performance according to the color variance $\sigma_{c}$.

For estimating the likelihoods $\boldsymbol{\theta}$ in (4), the pairwise affinity score $w_{n m}$ between two candidates $l_{n}=\left(x_{n}, x_{n^{\prime}}\right)$ and $l_{m}=\left(x_{m}, x_{m^{\prime}}\right)$ was defined under the basic and simple concept that a correspondence preserves adjacency relations in the image space, as follows.

$$
w_{n m}=\left\{\begin{array}{cc}
1 & \text { if }\left(x_{n}, x_{m}\right) \in \aleph \text { and }\left(x_{n^{\prime}}, x_{m^{\prime}}\right) \in \aleph^{\prime} \\
0 & \text { otherwise, }
\end{array}\right.
$$

where the adjacency relation $\left(x_{n}, x_{m}\right) \in \aleph\left(\right.$ or $\left.\left(x_{n^{\prime}}, x_{m^{\prime}}\right) \in \aleph^{\prime}\right)$ exists if regions $x_{n}$ (or $x_{n^{\prime}}$ ) and $x_{m}$ (or $x_{m^{\prime}}$ ) share a common boundary as in the work of Hedau et al. 4. We simply generated the seed weights $\hat{\boldsymbol{\eta}}$ for all correspondences $\bar{L}=L$ by using the 3 -dimensional color values as the feature property $v(\cdot)$ in (3). We initially set $\sigma_{w}=0.25 \mathrm{in}$ (3). We finally produced the optimal set $L^{\text {opt }}$ by Algorithm 1 without the optional step 4.

Fig. 2(c) and (d) compare the performance curves of our method with the 'Initial' method and the 'Spectral' method 7] as we varied the color noise $\sigma_{c}$ 


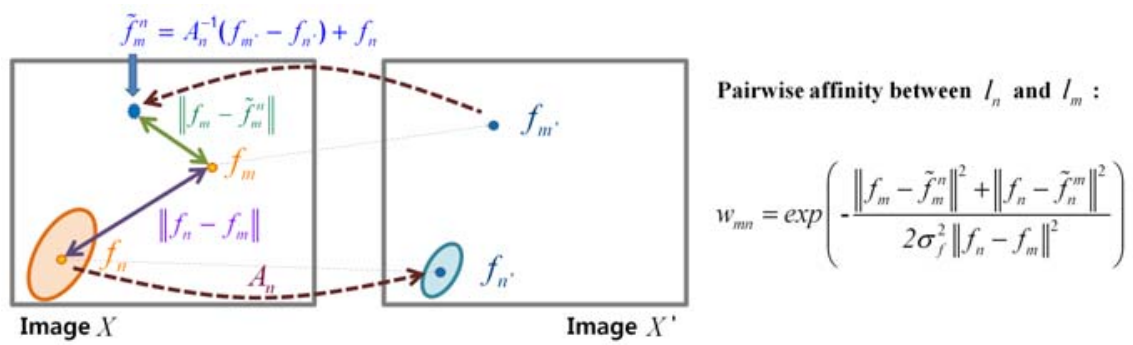

Fig. 3. Introducing our geometric affinity score $w_{n m}$ between two correspondences $l_{n}=\left(x_{n}, x_{n^{\prime}}\right)$ and $l_{m}=\left(x_{m}, x_{m^{\prime}}\right)$ for affine covariant features. Each feature $x_{n}$ has the $x-y$ position vector $f_{n}$. Each candidate $l_{n}$ has the local affine transformation matrix $\mathbf{A}_{n}$ between two features $x_{n}$ and $x_{n^{\prime}}$, estimated by [8] [10]. We initially set $\sigma_{f}=0.5$.

from 0 to 0.1 (in steps of 0.01 ), the number of matched regions $N^{p}$ from 16 to 25 . We compared the performances of these three methods over 30 trials by counting how many matches agreed with the ground truth. Note that the 'Initial' method is the same as Algorithm 1 with the seed weights $\hat{\boldsymbol{\eta}}$, instead of the likelihoods $\boldsymbol{\theta}$. It was included for testing the qualities of the seed weights $\hat{\boldsymbol{\eta}}$ itself. Although the pairwise affinity $w_{n m}$ in (6) for region matching only considers the adjacency relation, our method achieved better performance than other methods.

For discriminative features, the 'Spectral' method [7] uses a new weight matrix $\boldsymbol{\Pi}$, instead of $\mathbf{W}$, as follows.

$$
\boldsymbol{\Pi}=\mathbf{W}+\kappa \cdot \operatorname{diag}(\hat{\boldsymbol{\eta}}),
$$

where the parameter $\kappa$ controls the scale of the scores $\hat{\boldsymbol{\eta}}$ of each individual correspondence compared with the geometric affinity $\mathbf{W}$. With larger parameter $\kappa$, the discriminative power of each correspondence is more emphasized than pairwise relations. Fig. 2(e) shows that the performance of the 'Spectral' method is very sensitive to the parameter $\kappa$. We empirically set $\kappa=100$. Our method also needs one parameter: the restarting probability $\lambda$ in (4). However, compared with the 'Spectral' method, our method finds better correspondences with little performance changes according to the variation of $\lambda$, as shown in Fig. 2(f).

Interest Point Matching. We generally use the interest points, extracted by the affine covariant region detectors $8,10,12$, as features. We choose a candidate set $L$ of the $N$ feature correspondences with the condition that the descriptors are the nearest neighbors. Note that here the user could use different constraints for generating $L$. In this experiment, we used both affine covariant region detectors: the MSER detector [12] and the Hessian Affine detector [10] to obtain larger candidates $L$. The pairwise affinity $w_{n m}$ was defined as the spatial configuration between the neighboring feature correspondences, and based on the Euclidean distances between the pairs of features as shown in Fig. 3. This affinity matrix $\mathbf{W}$ well represents the pairwise geometric relationships under non-rigid deformations. The seed weights $\hat{\boldsymbol{\eta}}$ were obtained by using the SIFT 
descriptor [8] as the feature property $v(\cdot)$ in (3). We initially set $\sigma_{w}=0.2$ in (3). We finally produced the optimal set $L^{\text {opt }}$ by Algorithm 11 with the threshold $\gamma=0.9$.

Fig. 4 and Fig. 5 show the results of our feature matching in different dataset: ETHZ toys dataset and ICCV2005 datasets, respectively. These examples prove

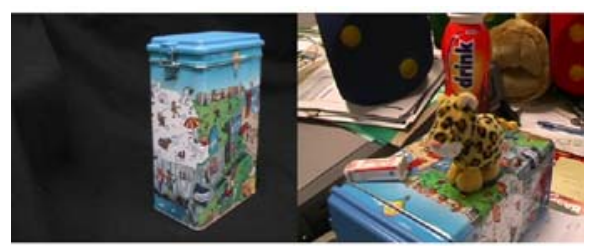

$|L|=707$

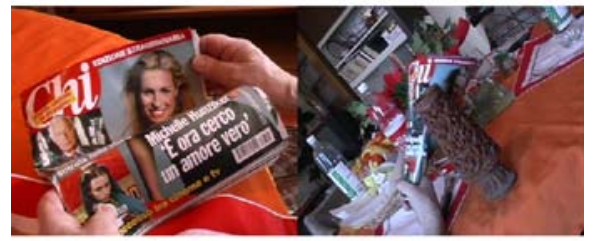

$|L|=1187$

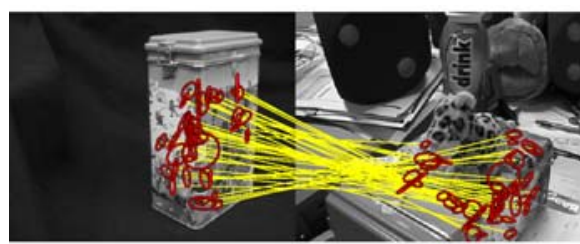

$\left|L^{o p t}\right|=34$

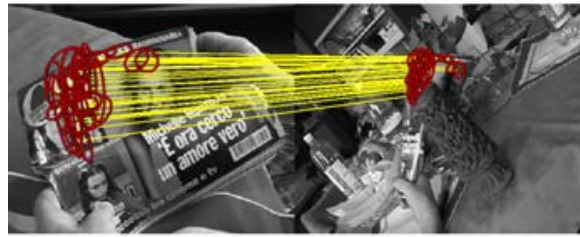

$\left|L^{o p t}\right|=30$

Fig. 4. Results of our feature matching in ETHZ toys dataset [1]: The original color images (the left column) and the matched features (right column)

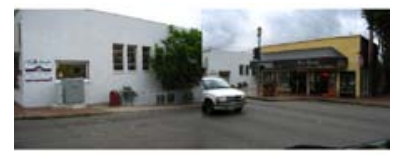

$L=\{42$ Inliers +279 Outliers $\}$

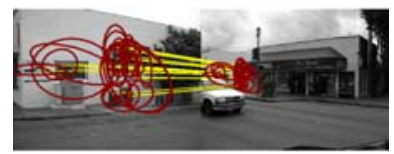

$L^{o p t}=\{25$ Inliers +0 Outliers $\}$

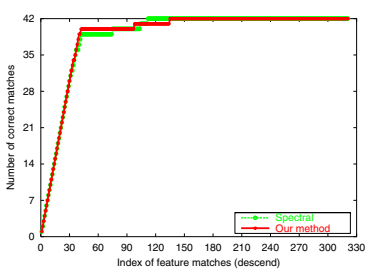

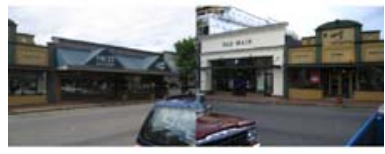

$L=\{17$ Inliers +324 Outliers $\}$

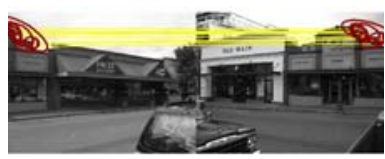

$L^{o p t}=\{9$ Inliers +0 Outliers $\}$

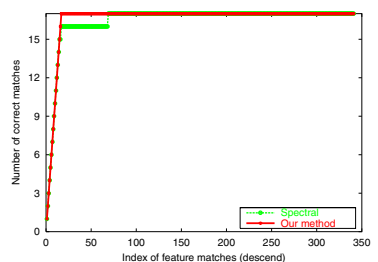

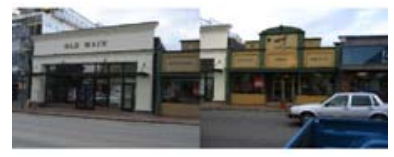

$L=\{41$ Inliers +355 Outliers $\}$

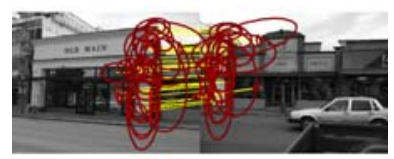

$L^{o p t}=\{15$ Inliers +0 Outliers $\}$

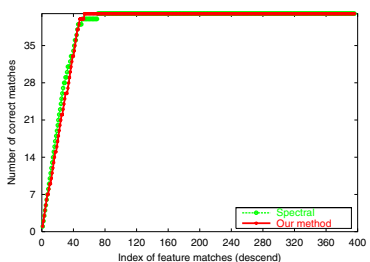

Fig. 5. Results of our feature matching between two scenes sharing overlapping fields of view in ICCV2005 datasets [2]. The matched features between each pair of the color images, displayed in the top row, are presented in the middle row. The graphs in the bottom row show the accumulated number of correct pairs in a high likelihood order. 
that our method produces well-matched pairs under difficult conditions such as nonrigid deformation and many outliers.

\section{Conclusions}

This paper presents a novel probabilistic framework for various correspondence problems. In this work, we design the likelihood of each correspondence as the weighted sum of all the RWR steady-state probabilities between feature correspondences. This likelihood efficiently represents the confidence of each candidate by considering the whole relations between two candidates in small neighborhood system. We finally obtain the optimal matches by a simple sequential method with mapping constraints. our method is qualitatively and quantitatively robust to noise and outliers in various matching frameworks. Our future work will include the candidate growing approach, instead of the fixed candidates $L$.

\section{Acknowledgement}

This research was supported in part by the IT R\&D program of MKE/IITA (2008-F-030-01), and in part by the Defense Acquisition Program Administration and Agency for Defense Development, Korea, through IIRC (UD070007AD).

\section{References}

1. ETHZ Toys Datatset, http://www.vision.ee.ethz.ch/ vferrari/datasets.html

2. ICCV2005 Dataset, http://research.microsoft.com/iccv2005/contest/

3. Berg, A.C., Berg, T., Malik, J.: Shape Matching and Object Recognition using Low Distortion Correspondences. In: CVPR, pp. 26-33 (2005)

4. Hedau, V., Arora, H., Ahuja, N.: Matching Images Under Unstable Segmentations. In: CVPR, pp. 1-8 (2008)

5. Kannala, J., Rahtu, E., Brandt, S.S., Heikkilä, J.: Object Recognition and Segmentation by Non-Rigid Quasi-Dense Matching. In: CVPR, pp. 1-8 (2008)

6. Kim, T.H., Lee, K.M., Lee, S.U.: Generative Image Segmentation Using Random Walks with Restart. In: Forsyth, D., Torr, P., Zisserman, A. (eds.) ECCV 2008, Part III. LNCS, vol. 5304, pp. 264-275. Springer, Heidelberg (2008)

7. Leordeanu, M., Hebert, M.: A Spectral Technique for Correspondence Problems Using Pairwise Constraints. In: ICCV, pp. 1482-1489 (2005)

8. Lowe, D.G.: Distinctive Image Features from Scale-Invariant Keypoints. IJCV 60(2), 91-110 (2004)

9. Maciel, J., Costeira, J.: A Global Solution to Sparse Correspondence Problems. PAMI 25(2), 187-199 (2003)

10. Mikolajczyk, K., Schmid, C.: Scale \& Affine Invariant Interest Point Detectors. IJCV 60(1), 63-86 (2004)

11. Pan, J.Y., Yang, H.J., Faloutsos, C., Duygulu, P.: Automatic Multimedia Crossmodal Correlation Discovery. In: KDD, pp. 653-658 (2004)

12. Tuytelaars, T., Gool, L.V.: Matching Widely Separated Views based on Affine Invariant Regions. IJCV 59(1), 61-85 (2004)

13. Zhou, D., Bousquet, O., Lal, T.N., Weston, J., Scholkopf, B.: Learning with Local and Global Consistency. In: NIPS, pp. 321-328 (2004) 